ИЗВЕСТИЯ АКАДЕМИИ НАУК ЭСТОНСКОИ ССР. ФИЗИКА * МАТЕМАТИКА PROCEEDINGS OF THE ACADEMY OF SCIENCES OF THE ESTONIAN SSR. PHYSICS * MATHEMATICS

$1989, \quad 38, \quad 2$

УДК 519.4

П. ПУУСЕМП

\title{
ОБ ОДНОЙ ТЕОРЕМЕ МЭЯ
}

\author{
(Представил Г. Вайникко)
}

\section{Введение}

C каждой группой $G$ можно связать совокупность End $G$ всех eе эндоморфизмов. Множество End $G$ образует полугруппу с нулем и единицей, где умножение понимается как суперпозиция отображений. Если группа $G$ абелева, то на множестве End $G$ можно определить еще сложение по правилу $g(x+y)=(g x)(g y)$, где $g \in G$ и $x, y \in$ End $G$. Тогда относительно этих двух действий множество End $G$ образует кольцо.

Теория колец эндоморфизмов абелевых групп хорошо развита. Один из центральных вопросов в этой теории следующий: когда из изоморфизма колец эндоморфизмов двух абелевых групп следует изоморфизм самих этих групп? Общеизвестна следующая теорема Бэра и Капланского (см. $\left[{ }^{1}\right]$, теорема 108.1): если $A$ и $B$ - периодические абелевы группы, ко́льца эндоморфизмов которых изоморфны, то всякий изоморфизм $\varphi$ между кольцами End $A$ и End $B$ индуцируется некоторым групповым изоморфизмом $\tau: A \rightarrow B$, т. е. $\varphi: \alpha \vdash \tau^{-1} \alpha \tau$ для каждого $\alpha \in$ End $A$. Аналогичный результат получил В. Мэй $\left[{ }^{2}\right]$. Сформулируем теорему Мэя. Пусть $G, G^{*}$ - абелевы группы и $G=A \oplus D$ - прямая сумма, где $A$ редуцирована и $D$ - непериодическая делимая группа, содержащая группы типа $p^{\infty}$ по всем простым числам $p$. Если группа $A$ вложима в группу $D$ и кольца эндоморфизмов групп $G$ и $G^{*}$ изоморфны, то всякий изоморфизм $\varphi$ между кольщами End $G$ и End $G^{*}$ индуцируется некоторым групповым изоморфизмом $\tau: G \rightarrow G^{*}$.

Полугруппам эндоморфизмов групп до сих пор уделяли мало внимания. Можно отметить лишь некоторые работы. Однако иногда полугруппа End $G$ хорошо характеризует группу $G$. Например, если полугруппа эндоморфизмов конечной абелевой группы $A$ изоморфна полугруппе эндоморфизмов некоторой другой группы $B$, то группы $A$ и $B$ изоморфны (см. $\left[{ }^{3}\right]$, теорема 4.2). В [ $\left.{ }^{4}\right]$ дано обобщение упомянутой теореме Бэра и Капланского. А именно, доказано, что если полугруппы всех эндоморфизмов двух периодических абелевых групп изоморфны, то сами эти группы изоморфны.

В настоящей работе дополним упомянутую теорему Мэя, т. е. докажем следующую теорему.

Т е о р е м. Пусть $G^{*}$ - некоторая группа, $A-$ редуцированная абелева группа и $D$ - непериодическая делимая абелева группа, содержащая группь типа ро для каждого простого числа р. Если

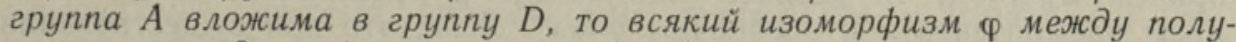
группами эндоморфизмов групп $G=A \oplus D u G^{*}$ индуцируется некоторым групповым изоморфизмом $\tau: G \rightarrow G^{*}$ (т. е. $\varphi: \alpha \longmapsto \tau^{-1} \alpha \tau$ при каждом $\alpha \in$ End $G$ ) и является также кольцевым изоморфизмом между End $G u$ End $G^{*}$. 
Кроме общепринятых обозначений будем придерживаться следующих: $I(G)$ - совокупность всех идемпотентных эндоморфизмов группы $G ; \mathbf{Q}$ - аддитивная группа рациональных чисел; $p$ - простое число; $Z\left(p^{\infty}\right)$ - группа типа $p^{\infty} ; P$ - совокупность всех простых чисел; End $G$ - полугруппа всех эндоморфизмов группы $G ; K_{G}(x)=$ $=\{y \in$ End $G \mid y x=x y=y\} ; \quad Q_{G}(x, y)=\{z \in$ End $G \mid x z=z y=z\}$. Отображение мы всегда пишем справа от элемента, на который оно действует. При изоморфизмах $G \cong \mathbf{Q}$ или $G \cong Z\left(p^{\infty}\right)$ мы часто для удобства проводим отождествление $G=\mathbf{Q}$ или $G=Z\left(p^{\infty}\right)$. Через $x^{*}$ всегда обозначен образ элемента $x \in \operatorname{End} G$ при изоморфизме End $G \cong$ $\cong$ End $G^{*}$. Для абелевых групп будем применять аддитивную запись.

\section{Вспомогательные леммы}

В настоящем и следующем параграфах всюду $G, G^{*}-$ произвольные группы. Перечислим некоторые известные результаты.

Л е м м а $1\left(\left[{ }^{3}\right]\right.$, лемма 1.1$)$. Если $x \in I(G)$, то группа $G$ разлагается в полупрямое произведение $G=\operatorname{Ker} x \lambda \operatorname{Im} x$ u $\operatorname{Im} x=\{g \in G \mid g x=$ $=g\}$.

Л е м м а $2\left(\left[{ }^{4}\right]\right)$. Пусть $x, y \in I(G)$. Тогда множество $Q_{G}(x, y)$ состоит из всевозможных эндоморфизмов $z$ группы $G$, для которых $z: \operatorname{Ker} x \rightarrow\langle 1\rangle$ u $z: \operatorname{Im} x \rightarrow \operatorname{Im} y$. Наоборот, каждый гомоморфизм $z: \operatorname{Im} x \rightarrow \operatorname{Im} y$ однозначно распространим на всю группу $G$ так, что $z: \operatorname{Ker} x \rightarrow\langle 1\rangle$.

Согласно лемме 2, мы можем отождествить всякий эндоморфизм $z \in Q_{G}(x, y)$ с соответствующим гомоморфизмом $z: \operatorname{Im} x \rightarrow \operatorname{Im} y$. Так как $Q_{G}(x, x)=K_{G}(x)$, то из леммы 2 следует

Л е м м а 3. Eсли $x \in I(G)$, то полугруппь $K_{G}(x) u$ End $(\operatorname{Im} x)$ изоморфны.

Следуя $\left[{ }^{5}\right]$ (с. 303), назовем эндоморфизмы $x$ и $y$ группы $G$ суммируемыми, если $g h=h g$ для каждых $g \in \operatorname{Im} x$ и $h \in \operatorname{Im} y$. Это равносильно тому, что сумма $x+y$ также является эндоморфизмом группы $G$. Эндоморфизмы $x$ и $y$ будем называть ортогональными, если $x y=y x=0$. В силу $\left[{ }^{3}\right]$ (с. 79,85 и 86 ), имеют место следующие две леммы.

Л е м м а 4. Если идемпотенты $x_{1}, \ldots, x_{n}$ nолугруппь End $G$ noпарно ортогональны $и$ суммируемы, то $x=x_{1}+\ldots+x_{n} \in I(G) u$

$\operatorname{Im} x=\operatorname{Im} x_{1} \times \ldots \times \operatorname{Im} x_{n} \quad$ (прямое произведение), $\operatorname{Ker} x=\bigcap_{i=1}^{n} \operatorname{Ker} x_{i}$.

Л ем м а 5. Eсли полугруппь End $G u$ End $G^{*}$ изоморфны $u$ $x_{1}, \ldots, x_{n}$ - попарно ортогональные и суммируемые идемпотенты из End $G$, то $x_{1}{ }^{*}, \ldots, x_{n}{ }^{*}$ также попарно ортогональны и суммируемы, причем

$$
\left(x_{1}+\ldots+x_{n}\right)^{*}=x_{1}^{*}+\ldots+x_{n}^{*} \text {. }
$$

Если $x, y$ - ортогональные и суммируемые идемпотенты из End $G$, причем $x+y=1$, то назовем идемпотент $y$ ортогональным дополнением идемпотента $x$ и обозначим $y=x^{\mathrm{T}}$. Ясно, что при существовании ортогонального дополнения оно определено однозначно и $G=$ $=\operatorname{Im} x^{\mathrm{T}} \times \operatorname{Im} x$. При этом $x, x^{\mathrm{T}}-$ проекции группы $G$ соответственно на подгруппы $\operatorname{Im} x$ и $\operatorname{Im} x^{\mathrm{T}}$. При изоморфизме End $G \cong$ End $G^{*}$ всегда $\left(x^{\mathrm{T}}\right)^{*}=\left(x^{*}\right)^{\mathrm{T}}$. 


\section{Свойства суммируемостй}

Предположим, что полугруппы End $G$ и End $G^{*}$ изоморфны. Тогда справедливы леммы 6-10.

Л ем м а 6. Если группы $G u G^{*}$ коммутативны; $x, y \in I(G) ; x, y-$ ортогональны $и$ и, $v \in Q_{G}(x, y)$, то $(u+v)^{*}=u^{*}+v^{*}$.

Д ок аз а тельство. Пусть выполнены предположения леммы. Тогда $x, y-$ суммируемы и согласно леммам 1 и 4

$$
\begin{aligned}
& G=(\operatorname{Ker} x \cap \operatorname{Ker} y) \oplus \operatorname{Im} x \oplus \operatorname{Im} y, \\
& \text { Ker } x=(\operatorname{Ker} x \cap \operatorname{Ker} y) \oplus \operatorname{Im} y, \\
& \text { Ker } y=(\operatorname{Ker} x \cap \operatorname{Ker} y) \oplus \operatorname{Im} x .
\end{aligned}
$$

По лемме $2 u: \operatorname{Im} x \rightarrow \operatorname{Im} y$. Поэтому существует единственный $w \in \operatorname{End} G$, обладающий свойствами

$$
\begin{array}{ll}
a w=a+a u, & a \in \operatorname{Im} x, \\
b w=b, & b \in \operatorname{Ker} x .
\end{array}
$$

Учитывая равенства (1), можно убедиться, что эндоморфизм ш характеризуется в полугруппе End $G$ свойствами

$$
x w x=x, \quad x w y=u, \quad x^{\mathrm{T}} w=x^{\mathrm{T}} .
$$

Аналогично существует единственный $t \in$ End $G$, обладающий свойствами

$$
x t x=x, \quad x t y=v, \quad x^{\mathrm{T}} t=x^{\mathrm{T}} .
$$

При таком $t$ имеем

$$
a t=a+a v, \quad b t=b
$$

H

$$
\begin{aligned}
& a(w t)=(a+a u) t=a+a v+a u=a+a(u+v), \\
& b(w t)=b,
\end{aligned}
$$

где $a \in \operatorname{Im} x, b \in \operatorname{Ker} x$. Следовательно, $u+v=x(w t) y$. Поэтому ясно, что $u+v$ полностью определяется действием умножения в полугруппе End $G$. В силу изоморфизма End $G \cong$ End $G^{*}$ сказанное выше переносится на полугруппу End $G^{*}$, т. е. имеет место равенство $(u+v)^{*}=$ $=u^{*}+v^{*}$. Лемма доказана.

Л ем м а 7. Пусть группы $G u G^{*}$ коммутативны; $x, y \in I(G)$; $x, y$ - ортогональны $u d \in Q_{G}(x, y)$, причем из $d u=d v ; u, v \in K_{G}(y)$, всегда следует равенство $u=v$. Тогда $(w+t)^{*}=w^{*}+t^{*}$ при кањдых $w, t \in K_{G}(y)$.

Д ок аз а тельст в о. Пусть выполнены предположения леммы и $w, t \in K_{G}(y)$. Тогда $d w, d t \in Q_{G}(x, y)$ и согласно лемме 6

$$
\begin{aligned}
& (d w+d t)^{*}=(d w)^{*}+(d t)^{*}=d^{*} w^{*}+d^{*} t^{*}=d^{*}\left(w^{*}+t^{*}\right), \\
& (d w+d t)^{*}=(d(w+t))^{*}=d^{*}(w+t)^{*} .
\end{aligned}
$$

Отсюда в силу изоморфизма End $G \cong$ End $G^{*}$ и предположений леммы следует равенство $(w+t)^{*}=w^{*}+t^{*}$. Лемма доказана.

Л е м м а 8. Если группы $G$ и $G^{*}$ коммутативны; $x, y \in I(G) ; x, y-$ ортогональны, $\operatorname{Im} x \cong \mathbf{Q}, \operatorname{Im} y \cong \mathbf{Q}$ или $\operatorname{Im} y \cong Z\left(p^{\infty}\right) u u, v \in K_{G}(y)$, то $(u+v)^{*}=u^{*}+v^{*}$.

Д оказ а тельство. Предположим, что выполнены предположения леммы. Так как $\mathbf{Q} / \mathbf{Q}_{p} \cong Z\left(p^{\infty}\right)$ (см. $\left[{ }^{6}\right]$, с. $27 ; \mathbf{Q}_{p}-$ множество всех рациональных чисел $m / n$, где число $n$ взаимно просто с числом 
$p)$, то существует такой $d \in Q_{G}(x, y)$, что $(\operatorname{Im} x) d=\operatorname{Im} y$. П̈оэтому всегда из равенства $d w=d t$, где $w, t \in K_{G}(y)$, следует равенство $w=t$. В силу леммы 7 имеем $(u+v)^{*}=u^{*}+v^{*}$. Лемма доказана.

Л емм а 9. Пусть $x, y_{1}, \ldots, y_{n} \in I(G) u y_{1}, \ldots, y_{n}-$ попарно ортогональны и суммируемы. Если $u_{i} \in Q_{G}\left(x, y_{i}\right)$ для каждого $i \in$ $\bar{E}\{1, \ldots, n\}$, то $\left(u_{1}+\ldots+u_{n}\right)^{*}=u_{1}^{*}+\ldots u_{n}^{*}$.

Доказ а тельство. Пусть выполнены предположения леммы. Можно считать, что $n=2$, ибо для произвольного $n$ можно доказательство провести индукцией по $n$. Итак, пусть $n=2$. Сумма $u_{1}+u_{2}$ равняется единственному эндоморфизму $w \in Q_{G}\left(x, y_{1}+y_{2}\right)$, удовлетворяющему условиям $w y_{1}=u_{1}$ и $w y_{2}=u_{2}$. Так как сказанное переносится на полугруппу End $G^{*}$ и по лемме $5\left(y_{1}+y_{2}\right)^{*}=y_{1}{ }^{*}+y_{2}{ }^{*}$, то $\left(u_{1}+u_{2}\right)^{*}=u_{1}^{*}+u_{2}^{*}$. Лемма доказана.

Л е м м а 10. Пусть $x, y_{1}, \ldots, y_{n} \in I(G)$ и $y_{1}, \ldots, y_{n}-$ попарно ортогональны и суммируемь. Если $K_{G}\left(y_{1}\right), \ldots, K_{G}\left(y_{n}\right)$ являются кольцами относительно умножения и сложения эндоморфизмов, и полугрупповой изоморфизм End $G \cong$ End $G^{*}$ индуцирует кольцевой изоморфизм $K_{G}\left(y_{i}\right) \cong K_{G}{ }^{*}\left(y_{i}{ }^{*}\right)$ для каждого $i \in\{1, \ldots, n\}$, то $(u+v)^{*}=$ $=u^{*}+v^{*} n р u \kappa a ж \partial ы х ~ u, v \in Q_{G}\left(x, y_{1}+\ldots+y_{n}\right)$.

Д ок а з тельство. Как и в предыдущей лемме, достаточно доказать настоящую лемму только при $n=2$. Пусть выполнены предположения леммы и $u, v \in Q_{G}\left(x, y_{1}+y_{2}\right)$. Тогда $u=u y_{1}+u y_{2}, v=$ $=v y_{1}+v y_{2} \quad$ и $u+v=\left(u y_{1}+v y_{1}\right)+\left(u y_{2}+v y_{2}\right)$. При этом $u y_{1}, v y_{1}$, $u y_{1}+v y_{1} \in Q_{G}\left(x, y_{1}\right)$ и $u y_{2}, v y_{2}, u y_{2}+v y_{2} \in Q_{G}\left(x, y_{2}\right)$. По лемме 9 и предположениям леммы имеем

$$
\begin{aligned}
(u+v)^{*} & =\left(u y_{1}+v y_{1}\right)^{*}+\left(u y_{2}+v y_{2}\right)^{*}= \\
& =\left(u y_{1}\right)^{*}+\left(v y_{1}\right)^{*}+\left(u y_{2}\right)^{*}+\left(v y_{2}\right)^{*}= \\
& =\left(u y_{1}\right)^{*}+\left(u y_{2}\right)^{*}+\left(v y_{1}\right)^{*}+\left(v y_{2}\right)^{*}= \\
& =\left(u y_{1}+u y_{2}\right)^{*}+\left(v y_{1}+v y_{2}\right)^{*}=u^{*}+v^{*} .
\end{aligned}
$$

Лемма доказана.

\section{Доказательство теоремы}

Фиксируем абелеву группу $G=A \oplus D$, где $A$ - редуцирована и вложима в группу $D$, а $D$ - непериодическая делимая группа, содержащая подгруппу $Z\left(p^{\infty}\right)$ для каждого простого числа $p$. Группа $D$ разлагается на прямую сумму $D=\bigoplus_{i \in I} D_{i}$, где $D_{i}$ изоморфна группе $\mathbf{Q}$ или группе $Z\left(p^{\infty}\right)$ для некоторого $p \in P$. Обозначим через $d$ некоторое вложение группы $A$ в группу $D$ и через $z, y, x_{i}, x_{J}$ проекции группы $G$ соответственно на подгруппы $D, A, D_{i}, \bigoplus_{i \in J} D_{i}(\varnothing \neq J \subset I)$. В силу предположений существуют такие $i_{0}, i_{p} \in I$, что $D_{i_{0}}=\mathbf{Q}$ и $D_{i_{p}}=Z\left(p^{\infty}\right)(p \in P)$. Обозначим $x_{i_{0}}=x, x_{i_{p}}=x_{p}$. Поэтому можно считать, что $P \subset I$.

Предположим теперь, что $G^{*}$ - некӧторая другая группа и End $G \cong$ End $G^{*}$ (изоморфизм полугрупп). Для доказательства теоремы покажем, что группа $G^{*}$ абелева и полугрупповой изоморфизм End $G \cong$ End $G^{*}$ является также кольцевым, т. е. $(u+v)^{*}=u^{*}+v^{*}$ для каждых $u$, $v \in$ End $G$. Тогда справедливость теоремы следует уже из упомянутой теоремы Мэя.

Так как $y, z$ - ортогональны, суммируемы и $y+z=1$, то $y^{*}, z^{*}-$ ортогональны, суммируемы и $y^{*}+z^{*}=1^{*}$. Поэтому $G^{*}=A^{*} \times D^{*}$, где 
$A^{*}=\operatorname{Im} y^{*}=\operatorname{Ker} z^{*}$ и $D^{*}=\operatorname{Im} z^{*}=\operatorname{Ker} y^{*}$. В силу леммы 3 End $D \cong$ End $D^{*}$. Следовательно,

$$
D \cong D^{*}, \quad D^{*}=\oplus_{i \in I} D_{i}^{*}, \quad D_{i}^{*}=\operatorname{Im} x_{i}^{*} \cong D_{i}=\operatorname{Im} x_{i}
$$

(см. $\left.\left[{ }^{7}\right]\right)$.

Л е м м а 11. Группа $A^{*}$ коммутативна.

Д ок аз а те льство. Так как $d \in Q_{G}(y, z)$, то $d^{*} \in Q_{G}{ }^{*}\left(y^{*}, z^{*}\right)$ и $\operatorname{Im} d^{*} \subset D^{*}$. Поэтому группа $\operatorname{Im} d^{*}$ коммутативна и коммутант группы $A^{*}$ содержится в $\operatorname{Ker} d^{*}$. Пусть $g \in A^{*}=\operatorname{Im} y^{*}$. Обозначим через $u^{*}$ такой элемент множества $K_{G}{ }^{*}\left(y^{*}\right)$, который действует на $A^{*}$ также как внутренний автоморфизм $g \wedge$, порожденный элементом $g$. Тогда при каждом $h \in G^{*}$ имеем

$$
\begin{gathered}
h\left(y^{*} u^{*} d^{*}\right)=\left(\left(h y^{*}\right) g^{\wedge}\right) d^{*}=\left(g^{-1} \cdot h y^{*} \cdot g\right) d^{*}= \\
=\left(h y^{*}\right)\left(\left(h y^{*}\right)^{-1} g^{-1}\left(h y^{*}\right) g\right) d^{*}=\left(h y^{*}\right) d^{*}=h\left(y^{*} d^{*}\right),
\end{gathered}
$$

т. е. $y^{*} u^{*} d^{*}=y^{*} d^{*}$ и $y u d=y d$. Поскольку $d-$ вложение группы $A=$ $=\operatorname{Im} y$ в группу $D$, то $y u=y$ и $y^{*} u^{*}=y^{*}$. Это означает, что $g \wedge$ действует тождественно на $A^{*}=\operatorname{Im} y^{*}$, т. е. группа $A^{*}$ коммутативна. Лемма доказана.

Уже доказано, что группа $G^{*}$ коммутативна.

Л е м м а 12. Если $u, v \in K_{G}(y)$, то $(u+v)^{*}=u^{*}+v^{*}$.

Доказ ательство. Пусть $u, v \in K_{G}(y)$. Тогда $u d, v d \in$ $\in Q_{G}(y, z)$ и в силу леммы 6

$$
(u d+v d)^{*}=(u d)^{*}+(v d)^{*}=u^{*} d^{*}+v^{*} d^{*}=\left(u^{*}+v^{*}\right) d^{*} .
$$

С другой стороны,

$$
(u d+v d)^{*}=((u+v) d)^{*}=(u+v)^{*} d^{*} .
$$

Поэтому $w^{*} d^{*}=0^{*}$, где $w^{*}=(u+v)^{*}-\left(u^{*}+v^{*}\right) \in K_{G}{ }^{*}\left(y^{*}\right)$. Тогда также $w d=0$ и $w=0$, ибо $w \in K_{G}(y)$ и $d-$ вложение группы $A=\operatorname{Im} y$ в $D$. Следовательно, $w^{*}=0^{*}$ и $(u+v)^{*}=u^{*}+v^{*}$. Лемма доказана.

Л е м а 13. Если $u^{*} \in K_{G}{ }^{*}\left(x^{*}\right) \quad$ и $u^{*} w^{*}=0^{*}$ для каждого $w^{*} \in$ $\in Q_{G}{ }^{*}\left(x^{*}, x_{P}{ }^{*}\right)$, то $u^{*}=0^{*}$.

Д ок а з а тельство. Предположим, что выполнены предположения леммы. В силу изоморфизма End $G \cong$ End $G^{*}$, тогда $u \in K_{G}(x)$ и $u w=0$ для каждого $w \in Q_{G}\left(x, x_{P}\right)$. Если $u \neq 0$, то существует такой $a \in \operatorname{Im} x=\mathbf{Q}$, что $a u \neq 0$, и можно указать $w \in Q_{G}\left(x, x_{P}\right)$ так, что $(a u) w \neq 0$, т. е. $u w \neq 0$. Действительно, в качестве $w$ можно взять произведение гомоморфизмов

$$
\mathbf{Q} \stackrel{\alpha}{\longrightarrow} \mathbf{Q} /\left\langle\frac{a u}{2}\right\rangle \stackrel{\beta}{\longrightarrow} \oplus_{p \in P} Z\left(p^{\infty}\right)=\operatorname{Im} x_{P}
$$

где $\alpha$ - естественный гомоморфизм и $\beta-$ некоторый изоморфизм (такой $\beta$ существует, см. $\left[{ }^{6}\right]$, с. 27 и 56). Следовательно, $u=0$ и $u^{*}=0^{*}$. Лемма доказана.

Л е м м а 14. Если $u, v \in K_{G}(x)$, то $(u+v)^{*}=u^{*}+v^{*}$.

Дока з а тельство. Предположим, что $u, v \in K_{G}(x)$. Пусть w - произвольный элемент из $Q_{G}\left(x, x_{P}\right)$. Тогда $w^{*}$ - произвольный элемент из $Q_{G^{*}}\left(x^{*}, x_{P}{ }^{*}\right) ; u w, v w \in Q_{G}\left(x, x_{P}\right) \quad$ и $\quad$ в

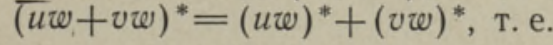

$$
\begin{gathered}
\left(\left(u^{*}+v^{*}\right)-(u+v)^{*}\right) w^{*}=u^{*} w^{*}+v^{*} w^{*}-(u+v)^{*} w^{*}= \\
=u^{*} w^{*}+v^{*} w^{*}-((u+v) w)^{*}=u^{*} w^{*}+v^{*} w^{*}-\left((u w)^{*}+(v w)^{*}\right)=0^{*}
\end{gathered}
$$


при каждом $w^{*} \in Q_{G}{ }^{*}\left(x^{*}, x_{P}{ }^{*}\right)$. Ввиду леммы 13 это́ озна́чает, что $\left(u^{*}+v^{*}\right)-(u+v)^{*}=0^{*}$, т. е. $(u+v)^{*}=u^{*}+v^{*}$. Лемма доказана.

Обозначим $U=\{y\} \cup\left\{x_{i} \mid i \in I\right\}$.

Л ем м а 15. Если $ш \in U \quad u \quad u, v \in K_{G}(w)$, то $(u+v)^{*}=u^{*}+v^{*}$.

Доказ ательство. Если $w=y$ или $w=x$, то утверждение следует из лемм 12 и 14, в противном случае из леммы 8. Лемма доказана.

Л е м м а 16. Если $u$, veEnd $G$, то $(u+v)^{*}=u^{*}+v^{*}$.

Д ок а з а т ел ь с т о. Предположим, что $u, v \in$ End $G$. Покажем равенство $(u+v)^{*}=u^{*}+v^{*}$. Для этого возьмем произвольный $g \in G^{*}$ и покажем, что $g(u+v)^{*}=g\left(u^{*}+v^{*}\right)$.

Так как

$$
G^{*}=A^{*} \oplus\left(\oplus_{i \in I} D_{i}^{*}\right)=\operatorname{Im} y^{*} \oplus\left(\oplus_{i \in I} \operatorname{Im} x_{i}^{*}\right),
$$

то существуют такие $y_{1}, \ldots, y_{n} \in U$, что элементы $g, g u^{*}, g v^{*}$ и $g(u+v)^{*}$ содержатся в $\operatorname{Im}\left(y_{1}{ }^{*}+y_{2}{ }^{*}+\ldots+y_{n}{ }^{*}\right)$. В силу леммы 1 идемпотент $y_{1}{ }^{*}+\ldots+y_{n}{ }^{*}=\left(y_{1}+\ldots+y_{n}\right)^{*}$ действует тождественно на элементы $g, g u^{*}, g v^{*}$ и $g(u+v)^{*}$. Следовательно,

$$
\begin{gathered}
g(u+v)^{*}=g\left(\sum_{j=1}^{n} y_{j}\right)^{*}(u+v)^{*}\left(\sum_{k=1}^{n} y_{k}\right)^{*}= \\
=g\left(\left(\sum_{j=1}^{n} y_{j}\right)(u+v)\left(\sum_{k=1}^{n} y_{k}\right)\right)^{*}=g\left(\sum_{j, k=1}^{n}\left(y_{j} u y_{k}+y_{j} v y_{k}\right)\right)^{*} .
\end{gathered}
$$

При этом $y_{j} u y_{k}, y_{j} v y_{k} \in K_{G}\left(y_{1}+\ldots+y_{n}\right)$. Ввиду лемм 10 (взять там $\left.x=y_{1}+\ldots+y_{n}\right)$ и 15 ясно, что изоморфизм End $G \cong$ End $G^{*}$ индуцирует изоморфизм колец $K_{G}\left(y_{1}+\ldots y_{n}\right)$ и $K_{G}{ }^{*}\left(y_{1}{ }^{*}+\ldots+y_{n}{ }^{*}\right)$. Поэтому в силу равенства (2) имеем

$$
\begin{aligned}
g(u+v)^{*} & =g\left(\sum_{j, k=1}^{n}\left(\left(y_{j} u y_{k}\right)^{*}+\left(y_{j} v y_{k}\right)^{*}\right)\right)= \\
& =g\left(\sum_{j, k=1}^{n}\left(y_{j}{ }^{*} u^{*} y_{k}{ }^{*}+y_{j}{ }^{*} v^{*} y_{k}{ }^{*}\right)\right)= \\
& =g\left(\sum_{j=1}^{n} y_{j}^{*}\right)\left(u^{*}+v^{*}\right)\left(\sum_{k=1}^{n} y_{k}{ }^{*}\right)=g\left(u^{*}+v^{*}\right) .
\end{aligned}
$$

Лемма доказана.

Теорема доказана.

Непосредственно из этой теоремы вытекает

Сле дствие. Для каждой абелевой группь А существует делимая абелева группа $D$ так, что прямая сумма $A \oplus D$ определяется ее полугруппой эндоморфизмов в классе всех групп.

\section{ЛИТ Е РА Т Р А}

1. Фукс Л. Бесконечные абелевы группы. М., Мир, 1977. Т. 2.

2. May, W. // Bull. London Math. Soc., 1978, 10, № 3, 270-272.

3. Пуусемп П. // Уч. зап. Тартуск. ун-та, 1975, № 366, 76-104.

4. Пуусемп П. // Изв. АН ЭССР. Физ. Матем., 1980, 29, № 3, 246-253.

5. Курош А. Г. Теория групп. М., Наука, 1967.

6. Фукс Л. Бесконечные абелевы группы. Т. 1. М., Мнр, 1974.

7. Пуусемп П. // Тез. докл. конф. «Методы алгебры и анализа». Тарту, 1983, 14-16.

Таллиннский политехнический институт

Поступила в редакцию 16/II 1988 


\section{Pि. PUUSEMP̉}

\section{OHEST MAY TEOREEMIST}

\section{On tõestatud}

T e o reem. Olgu $H$ suvaline rühm, $A$ redutseeritud kommutatiivne rühm ja $D$ mitteperioodiline jaguv kommutatiivne rühm, mis sisaldab iga algarvu $p$ jaoks jaguvat rühma $Z\left(p^{\infty}\right)$. Kui rühm $A$ on sisestatav rühma $D$, siis rühmade $G=A \oplus D$ ja $H$ endomorfismipoolrühmade iga isomorfism $\varphi$ indutseeritakse isomorfismiga $\tau: G \rightarrow H$ (s.t. $\varphi: \alpha \rightarrow \tau^{-1} \alpha \tau$ iga $\alpha \in$ End $G$ korral) ning isomorfism $\varphi$ on ka rühmade $G$ ja $H$ endomorfismiringide isomorfism.

See teoreem üldistab May teoreemi $\left[{ }^{2}\right]$. Tōestatud teoreemist järeldub vahetult

$\mathrm{J}$ ä reldus. Iga kommutatiivse rühma A jaoks leidub selline jaguv kommutatiivne rühm $D$, et otsesumma $A \oplus D$ on määratud oma endomorfismipoolrühmaga kōikide rühmade klassis.

\section{P. PUUSEMP}

\section{ON A MAY THEOREM}

All the endomorphisms of an Abelian group $G$ form a ring. The Abelian group $G$ is characterized by this ring. For example, it is well known that the isomorphism of two periodic Abelian groups $G$ and $H$ follows from the isomorphism of rings of all endomorphisms of $G$ and $H$. All the endomorphisms of an arbitrary group $G$ form only a semigroup End $G$. If, from the isomorphism of semigroups End $G$ and End $H$ of groups $G$ and $H$ always follows the isomorphism of groups $G$ and $H$, then we say that the group $G$ is determined by its semigroup of endomorphisms (in the class of all groups). In general, the necessary and sufficient conditions are not known for determining an arbitrary group by its semigroup of endomorphisms. Some results concerning this problem are presented in the papers $[3,4,7]$. For example, in the paper $\left[{ }^{3}\right]$ it is shown that from the direct decomposition $G=G_{1} \times \ldots \times G_{n}$ and the isomorphism End $G \cong$ End $H$ the direct decomposition $H=H_{1} \times \ldots \times H_{n}$ follows with a property End $G_{i} \cong$ End $H_{i}$ for any $i \in\{1, \ldots, n\}$. From this result it is deduced in a simple way that an arbitrary finite Abelian group can be determined by its semigroup of endomorphisms (see [ $\left.{ }^{3}\right]$, theorem 4.2).

In this paper, using the methods and results of the papers $\left[{ }^{2,3}\right]$, the following theorem is proved.

Theorem. Let $G=A \oplus D$, where $D$ is a non-periodic divisible Abelian group that contains g group $Z\left(p^{\infty}\right)$ for any prime number $p$ and $A$ is a reduced Abelian group that is embeddable in the group $D$. If $H$ is an arbitrary group, and if $\Phi$ : End $G \rightarrow$ End $H$ is an isomorphism of semigroups End $G$ and End $H$, then there exists an isomorphism $\varphi: G \rightarrow H$ such that $\alpha \Phi=\varphi^{-1} \alpha \varphi$ for every $\alpha \in$ End $G$ and $\Phi$ is also the isomorphism for rings of endomorphism of the groups $G$ and $H$. follows

This theorem generalizes a May theorem (see $\left[{ }^{2}\right]$, theorem). From this theorem

Corollary. For an arbitrary Abelian group $A$ there exists a divisible Abelian group $D$ such that the direct sum $A \oplus D$ is determined by its semigroups of endomorphisms in the class of all groups. 